\title{
Experimental verification of homogeneity in polymer gels
}

\author{
Takamasa Sakai
}

The realization of an ideal polymer network that is free from heterogeneity is one of the ultimate goals in polymer science. Heterogeneity is known to be an inherent property of polymer networks. To realize an ideal polymer network, we need to develop methods to achieve a homogeneous network structure and to confirm the network homogeneity. This is a dilemma, as the former method needs to be verified by the latter method, while the latter method needs to be examined by the former method. To resolve this situation, a series of studies on polymer networks with well-defined properties is needed. We recently designed and fabricated novel Tetra-polyethyleneglycol (PEG) hydrogels. Characterization by small angle neutron scattering, infrared spectroscopy, nuclear magnetic resonance and tearing experiments indicates that Tetra-PEG gels have highly suppressed heterogeneity. In this review, we show a series of experimental results from Tetra-PEG gels and discuss the results in terms of network homogeneity. Finally, using Tetra-PEG gels as a model system, we examine models for predicting the elastic modulus of polymer gels and show that Tetra-PEG gels are useful as a model system for evaluating models that predict the physical properties of polymer networks.

Polymer Journal (2014) 46, 517-523; doi:10.1038/pj.2014.28; published online 28 May 2014

Keywords: heterogeneity; ideal polymer network; polymer gels; Tetra-PEG gel

\section{INTRODUCTION}

Polymer gels consist of three-dimensional (3D) polymer networks that are swollen in solvent. Because the polymer chains in 3D polymer networks are connected by cross-links, the structure of gels is distinctly different compared with that of uncross-linked polymer melts and polymer solutions. 3D polymer networks are inherently heterogeneous. This heterogeneity results from transient heterogeneity in the polymer chains, which normally decays over a finite period of time but becomes immobilized during cross-linking. Heterogeneity in $3 \mathrm{D}$ polymer networks is categorized based on size as being either global or local (Figures 1a and b). Global heterogeneity, which is also called spatial heterogeneity, is the heterogeneous distribution of polymer segments on the order of tens of nanometers to several micrometers. ${ }^{1-3}$ Spatial heterogeneity is detected as excess scattering in a variety of scattering analyses. In contrast, local heterogeneity is the result of defects formed by a small number of chains, such as trapped entanglements and connectivity defects (Figures $1 \mathrm{c}$ and d). ${ }^{4-6}$ Local heterogeneity is difficult to detect and is detected as a discrepancy in physical properties compared with model predictions. Importantly, before the detection of heterogeneity, such models need to be validated using homogenous polymer networks (that is, ideal polymer networks). However, the homogeneity of a polymer network cannot be verified without knowing the correct model. To overcome this dilemma, a series of studies on polymer networks with controlled network structures is needed. The most popular approach for realizing controlled polymer network structures is 'the model network system, in which $3 \mathrm{D}$ polymer networks are formed from telechelic prepolymers and multi-functional crosslinkers. $^{7-9}$ Because telechelic prepolymers have a defined degree of polymerization and multi-functional crosslinkers have a defined functionality, the achievement of a homogeneous polymer network is expected. However, even in these model networks, excess scattering and systematic disagreement between predicted and experimental physical properties have been observed, ${ }^{10,11}$ suggesting the existence of global and local heterogeneity.

Recently, we improved the model network system and designed Tetra-polyethyleneglycol (PEG) gels. ${ }^{12-21}$ Tetra-PEG gels are formed from mutually reactive tetra-armed PEG prepolymers in an aqueous solution (AB-type crosslink coupling, Figure 1e). The prepolymers have amine and activated ester end groups, resulting in linking through amide bonds. In this system, the reaction rate is controlled by tuning the $\mathrm{pH}$ of the aqueous solution. ${ }^{17,19}$ As far as we know, the Tetra-PEG gel system offers three advantages over conventional model network systems. The first advantage is rate control over reaction conversion, which allows for the homogeneous mixing of prepolymers. The second advantage is the presence of solvent. Solvent gives fluidity to the pregel solution and further contributes to the homogenous mixing of the two prepolymers. Solvent also prevents the overlapping of chains, which leads to the inhibition of trapped entanglements. The third advantage is the shape of the 

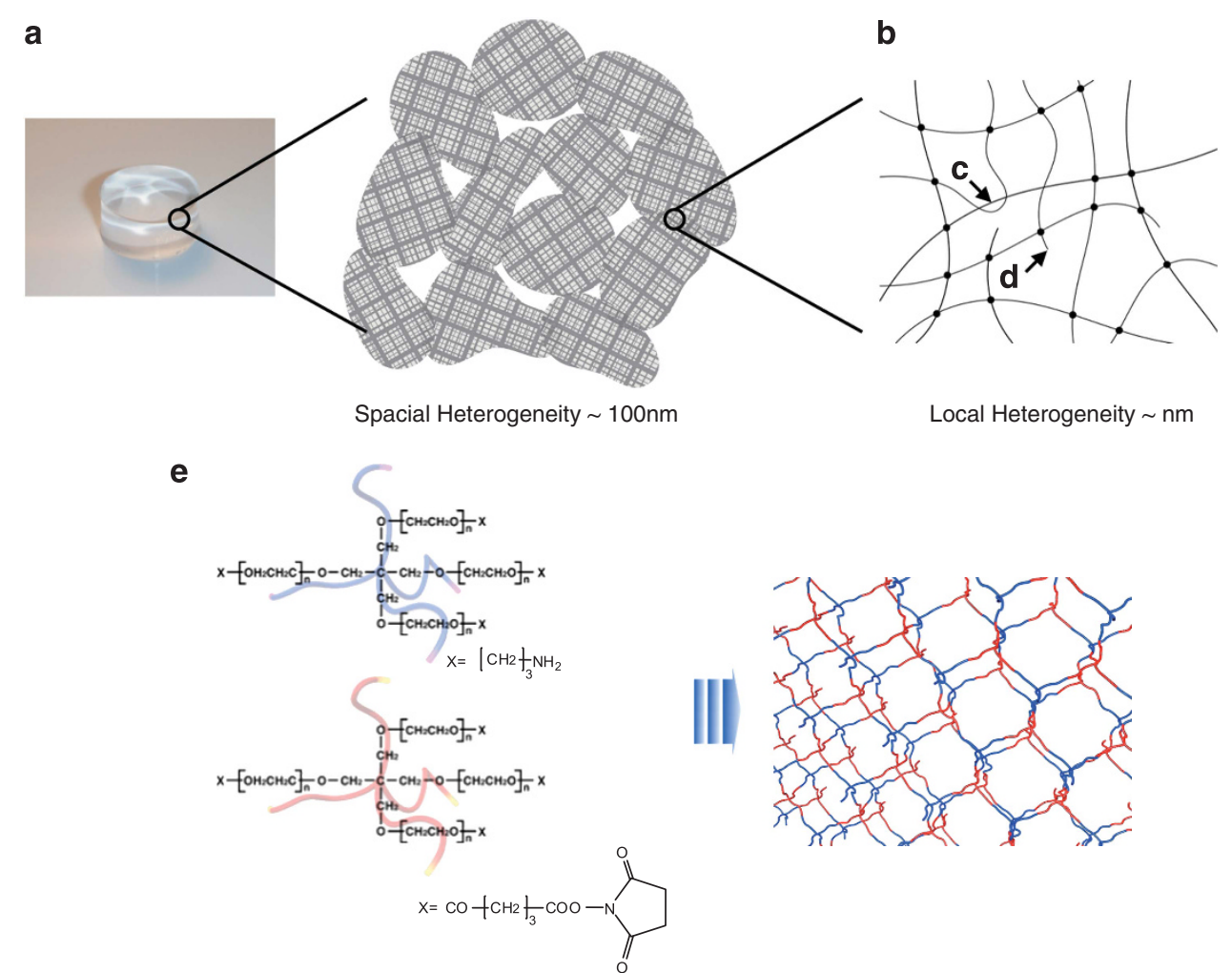

Figure 1 Heterogeneity of polymer gels categorized into (a) spatial heterogeneity $(\sim 100 \mathrm{~nm})$ and (b) local heterogeneity $(\sim \mathrm{nm})$. Local heterogeneity includes (c) trapped entanglements, (d) dangling ends and other connectivity defects. (e) Schematic illustration of tetra-polyethyleneglycol gel.

prepolymers, which does not allow the formation of back-biting loop structures. Collectively, these advantages impart homogeneity to the Tetra-PEG gel network structure. We formed Tetra-PEG gels under a variety of conditions and investigated their global and local structures, and we found that Tetra-PEG gels have a homogeneous network structure under appropriate conditions. In this review, we present a series of experimental results and discuss the homogeneity of Tetra-PEG gels.

\section{SPATIAL HETEROGENEITY}

Spatial heterogeneity is the static density fluctuation in polymer gels and is the most common heterogeneity in polymer gels. This heterogeneity is observed as excess scattering in small angle neutron scattering and static light scattering measurements, and the size scale is from several tens of nanometers to several micrometers. The scattering curves of conventional polymer gels are represented by the superposition of the Ornstein-Zernike function, ${ }^{22}$ which corresponds to scattering from the homogeneous polymer solution, and the squared Lorentz or Gauss functions, which are representative of the excess scattering: 5,23

$$
\begin{aligned}
& I(q)=\frac{(\Delta \rho)^{2} R T \phi^{2}}{N_{\mathrm{A}} M_{\mathrm{os}}} \frac{1}{1+\xi^{2} q^{2}} \\
& I(q)=\frac{(\Delta \rho)^{2} R T \phi^{2}}{N_{\mathrm{A}} M_{\mathrm{OS}}} \frac{A_{\text {hetero }}}{\left(1+\Xi^{2} q^{2}\right)^{2}}
\end{aligned}
$$

where $(\Delta \rho)^{2}$ is the scattering length density difference square between the polymer and solvent, $R$ is the gas constant, $T$ is the absolute temperature, $N_{\mathrm{A}}$ is Avogadro's number, $\varphi$ is the polymer volume fraction, $M_{\mathrm{OS}}$ is the longitudinal modulus, $\xi$ is the correlation length of the network, $\Xi$ is the characteristic size of heterogeneities in the gel, and $A_{\text {hetero }}$ is a constant representing the contribution of frozen heterogeneity and is independent of $q$. In contrast, the scattering curve of Tetra-PEG gels is reproduced only by the Ornstein-Zernike function in the region $<200 \mathrm{~nm}^{24}$ Although excess scattering was observed in the region $>200 \mathrm{~nm}$, the $q$-dependence of excess scattering of Tetra-PEG gels $\left(I(q) \sim q^{-2}\right)$ is much lower than that of conventional polymer gels $\left(I(q) \sim q^{-4}\right)$. In addition, even in the equilibrium-swollen state, where spatial heterogeneity is emphasized, no appreciable excess scattering was observed ${ }^{25}$ (Figure 2). These data strongly suggest highly suppressed spatial heterogeneity in Tetra-PEG gels. The near-absence of spatial heterogeneity allows us to investigate the relationship between physical properties and structures in the following sections.

\section{CONNECTIVITY HETEROGENEITY}

When we focus on the local connectivity of the polymer network, we can imagine substructures including the perfect crosslink (Figure 3b), an unreacted end (Figure 3c), double links (Figure 3d), triple links (Figure $3 \mathrm{e}$ ) and so on, where substructures other than perfect crosslinks are connectivity defects. Among these substructures, unreacted ends are the primary source of connectivity heterogeneity. We can estimate the fraction of unreacted ends from the reaction conversion $(p)$. Using infrared spectroscopy, we directly measured the concentrations of unreacted groups ([carboxyl]) and reacted groups ([amide]), and estimated $p$ as $p=[$ amide $] /([$ amide $]+[$ carboxyl $]$ ) (Figure $3 a) .{ }^{13}$ We tuned the molecular weights $(M)$ and initial polymer volume fraction $\left(\phi_{0}\right)$ of the prepolymers. The values of $p$ were up to 0.9 , and no appreciable dependence on $\phi_{0}$ was observed. It should be noted that $p$ was almost constant below and above the overlap concentration of the prepolymers $\left(\phi^{*}\right)$. Thus, we can achieve high-reaction conversion up to 0.9 , even below $\phi^{*}$. Furthermore, our analysis of 


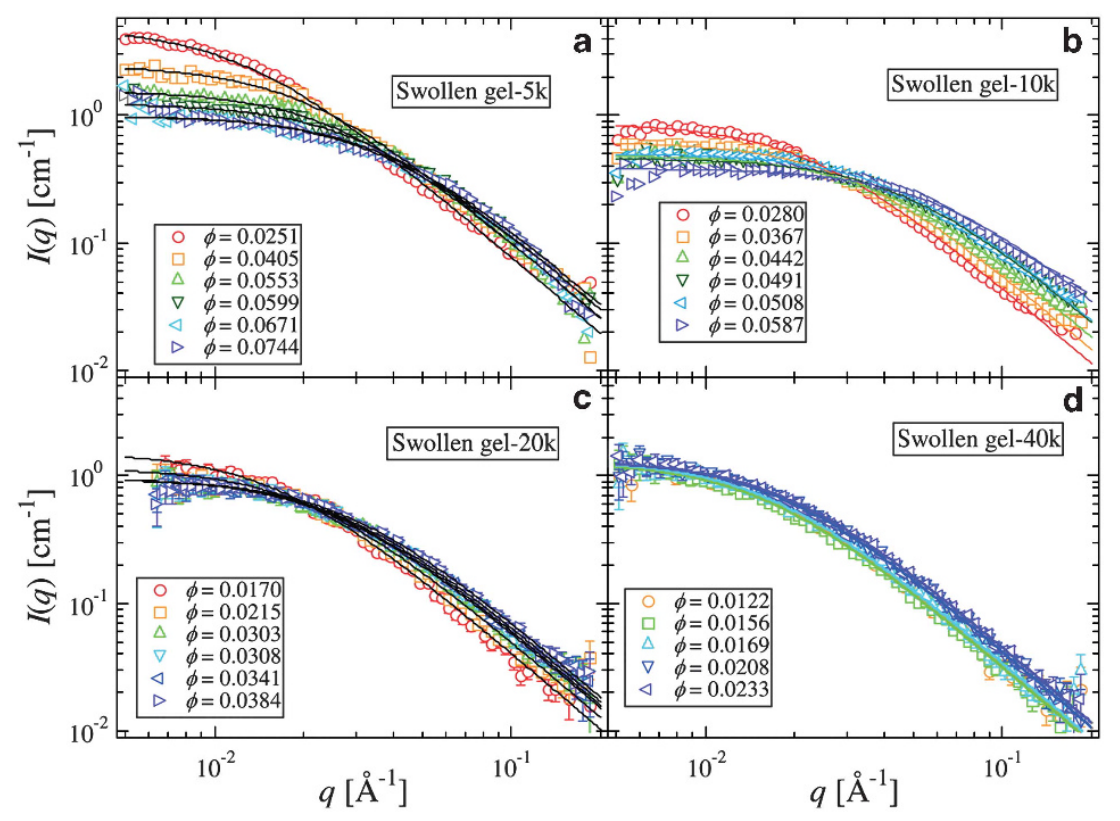

Figure 2 Series of SANS intensity functions for tetra-polyethyleneglycol (PEG) gels at swelling equilibrium for (a) 5k (b) 10k, (c) 20k and (d) 40k tetra-PEG gels. The solid lines denote the results of curve fitting with Ornstein-Zernike functions (reproduced from Matsunaga et al. ${ }^{24}$ with permission from the American Chemical Society).

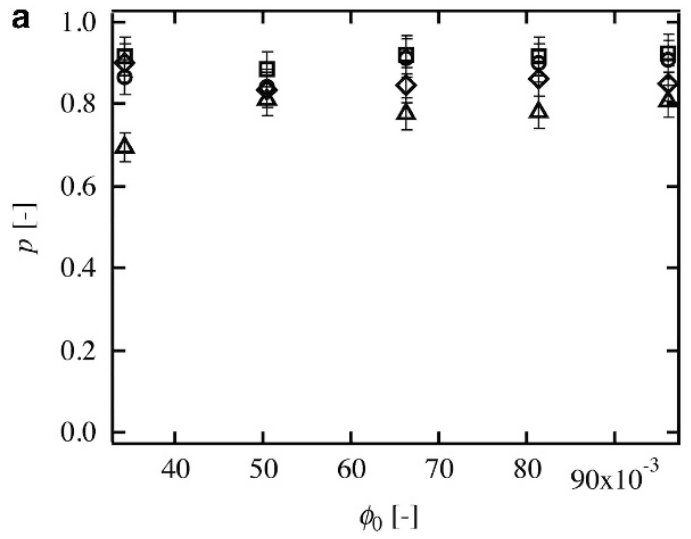

b Perfect $x$-link

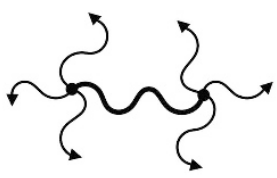

d Double link

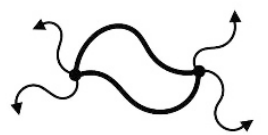

C Unreacted end

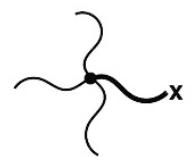

e Triple link

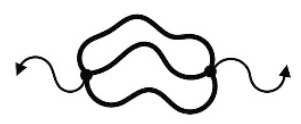

Figure 3 (a) Reaction conversion ( $p$ ) of $5 \mathrm{k}, 10 \mathrm{k}, 20 \mathrm{k}$ and $40 \mathrm{k}$ tetrapolyethyleneglycol gels. Schematic pictures of local connectivity: (b) perfect crosslink, (c) an unreacted end, (d) double links and (e) triple links. Arrows indicate that these arms lead out to the macroscopic body (reproduced from Akagi et al. ${ }^{30}$ with permission from the American Chemical Society).

the reaction kinetics revealed that this reaction proceeds as a second order reaction of amine and activated ester with a constant reaction rate from initiation to completion, regardless of the gelation

threshold. These results suggest that the prepolymers are premixed homogeneously and react efficiently.

In addition, we investigated the higher order connectivity heterogeneity (Figures $3 \mathrm{~d}$ and e) by ${ }^{1} \mathrm{H}$ multiple-quantum nuclear magnetic resonance spectroscopy. ${ }^{26} \mathrm{We}$, for the first time, observed quantized spectra originating from different orders of connectivity heterogeneity. The fraction of prepolymers with perfect cross-links was up to 0.7. The fractions of prepolymers with defects corresponding to unreacted ends or other structures were 0.1 and 0.2 , respectively. Although there is connectivity heterogeneity in Tetra-PEG gels, the degree of heterogeneity is highly suppressed compared with other polymer gels. This series of studies is the first experimental detection and categorization of the connectivity heterogeneity of polymer gels.

\section{ELASTICALLY EFFECTIVE CHAINS}

To predict the mechanical properties of Tetra-PEG gels, we need to distinguish whether a strand is elastically effective. An elastically effective chain is defined as a strand connecting two active crosslinks. ${ }^{27,28}$ An active crosslink is defined as a junction point having more than three connections to the macroscopic body and is assumed to deform in the same manner as the macroscopic deformation. It should be noted that an arm being connected to the macroscopic body is different from an arm being connected to the next arm; the former case includes the latter case, but the opposite is not true. Miller and Macosko ${ }^{29}$ applied a tree-like approximation to estimate the connectivity of an arm to the macroscopic body. Their model was developed based on the recursive nature of the branching process and on elementary probability laws with the following three assumptions: (1) all functional groups of the same type are equally reactive, (2) all groups react independently of one another and (3) no intramolecular reactions occur in finite species. The Tetra-PEG gel system is considered an AB-type stepwise copolymerization of tetrafunctional prepolymers $\left(\mathrm{A}_{4}\right.$ and $\left.\mathrm{B}_{4}\right)$. Under the stoichiometric 
condition of $\mathrm{A}_{4}$ and $\mathrm{B}_{4}$, this reaction is reduced to a stepwise homopolymerization of $\mathrm{A}_{4}$. Here, we consider the probability that one of the four arms leads out to a 'finite' chain $\left(P\left(F^{\text {out }}\right)\right)$, which corresponds to one of the four arms leading out to the macroscopic body. $P\left(F^{\text {out }}\right)$ is given by the sum of the probabilities that an arm is reacted to the next arm and then three of four arms on the next prepolymer leads out to a 'finite' chain, and that an arm does not react with the next arm:

$$
P\left(F^{\text {out }}\right)=p \cdot P\left(F^{\text {out }}\right)^{3}+(1-p)
$$

where $p$ is the reaction efficiency. By solving this equation, we obtain

$$
P\left(F^{\text {out }}\right)=\left(\frac{1}{p}-\frac{3}{4}\right)^{\frac{1}{2}}-\frac{1}{2} .
$$

Using $P\left(F^{\text {out }}\right)$, the probability that a given tetra-arm polymer belongs to active cross-links through three and four arms $\left(P\left(X_{3}\right)\right.$ and $\left.P\left(X_{4}\right)\right)$ is represented as

$$
\begin{aligned}
& P\left(X_{3}\right)={ }_{4} C_{3} \cdot P\left(F^{\text {out }}\right) \cdot\left[1-P\left(F^{\text {out }}\right)\right]^{3} \\
& P\left(X_{4}\right)=\left[1-P\left(F^{\text {out }}\right)\right]^{4} .
\end{aligned}
$$

The concentrations of cross-links $(\mu)$ and elastically effective chains
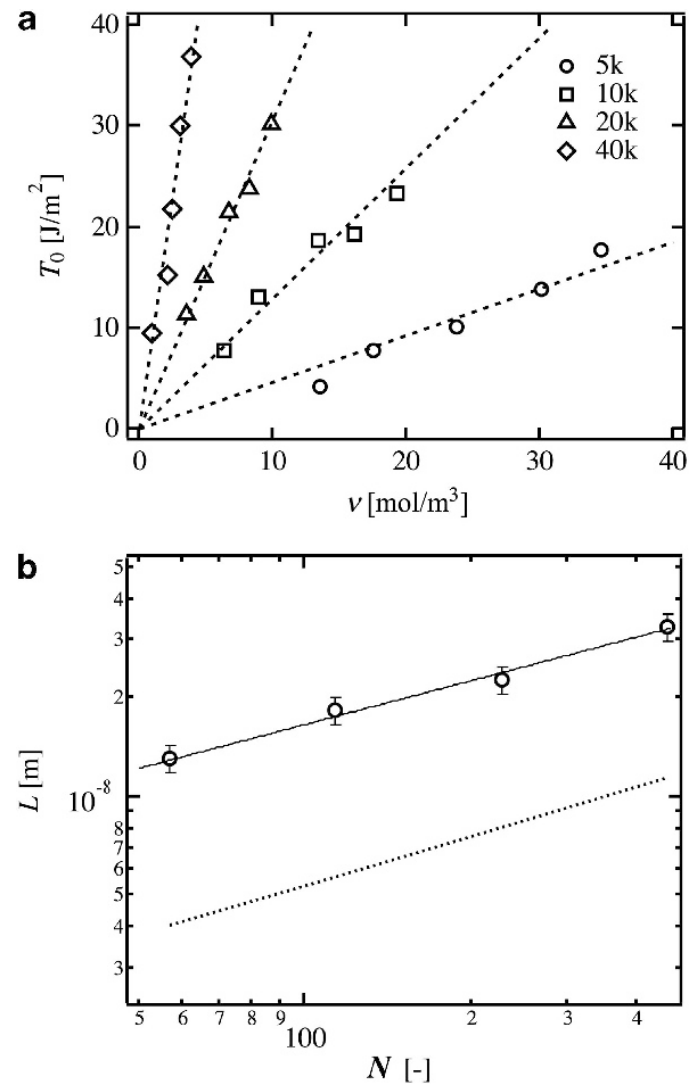

Figure 4 (a) $T_{0}$ as a function of $v$ in the tetra-polyethyleneglycol (PEG) gels (5k, rhombus; $10 \mathrm{k}$, circle; $20 \mathrm{k}$, square; $40 \mathrm{k}$, triangle). Linear fitting of the data is shown as dotted lines. (b) $L$ as a function of $N$ in tetra-PEG gels. The solid line corresponds to a fit line for a power function $\left(L \sim N^{0.45}\right)$, and the dashed line is the guide showing the computed $R_{0}$ of network chains with polymerization degrees of $N$ (reproduced from Akagi et al..$^{31}$ with permission from the American Institute of Physics). $(v)$ are represented as follows,

$$
\begin{aligned}
& \mu=c \cdot\left(P\left(X_{3}\right)+P\left(X_{4}\right)\right) \\
& v=c \cdot\left(\frac{3}{2} \cdot P\left(X_{3}\right)+2 \cdot P\left(X_{4}\right)\right) .
\end{aligned}
$$

where $c$ represents the molar concentration of the tetra-arm polymer in the unit volume of material. Using Equations 3-8, we can estimate $\mu$ and $v$ from the value of $p$.

\section{TRAPPED ENTANGLEMENTS}

Because trapped entanglements cannot be undone, even after a long period of time, these defects influence the mechanical properties. In the case of tearing, trapped entanglements are expected to act as pseudo-cross-links because the polymer chains in the vicinity of a crack tip are fully stretched. ${ }^{28}$ Thus, we performed tearing tests on trouser-shaped specimens to investigate trapped entanglements. ${ }^{30,31}$ Each specimen was pulled at a constant speed $\left(500 \mathrm{~mm} \mathrm{~min}^{-1}\right)$, and the tearing force $(F)$ was recorded. The fracture energy $\left(T_{0}\right)$ is given as ${ }^{32}$

$$
T_{0}=\frac{2 F}{h}
$$

where $h$ is the thickness of the gel sample. The tearing force $F$ fluctuated during the experiment, showing stick-slip-like behavior. We computed $T_{0}$ from the minimum values of $F$, which indicates the minimum energy required to propagate a crack. The obtained $T_{0}$ corresponded to the equilibrium values without time effects, as no appreciable difference was noted for different crosshead speeds.

The most popular model for predicting $T_{0}$ of elastomeric materials is the Lake-Thomas model. ${ }^{33}$ The Lake-Thomas model describes $T_{0}$ as the energy needed to break the chemical bonds per unit crosssection on the fracture surface as

$$
T_{0}=\left(\frac{3}{8}\right)^{1 / 2} v L N U
$$

where $L$ is the displacement length, $N$ is the degree of polymerization of network strands and $U$ is the energy required to rupture a monomer unit. This model assumes that $L$ is related to the end-toend distance of the network strand according to $L \approx R_{0} \approx a N^{1 / 2}$ (a, monomer length) and that $U$ is the sum of the bond energies in a monomeric unit. According to the Lake-Thomas model, two scaling relationships are expected for polymer gels without trapped entanglements: $T_{0} \sim v$ and $L \sim N^{1 / 2}$. However, for polymer gels with trapped entanglements, the scaling relationships change to $T_{0} \sim(v+\varepsilon)$ and $L \sim N_{\mathrm{e}}{ }^{1 / 2}$, where $\varepsilon$ is the number density of pseudo-cross-links and $N_{\mathrm{e}}$ is the degree of polymerization of neighboring cross-links or pseudo-cross-links.

We estimated $T_{0}$ for $5 \mathrm{k}, 10 \mathrm{k}, 20 \mathrm{k}$ and $40 \mathrm{k}$ Tetra-PEG gels with different values of $\phi_{0}$ and plotted these values against $v$ estimated from a tree-like approximation (Figure 4a). The change in $v$ for each symbol is derived from a change in $\phi_{0}$. For each Tetra-PEG gel, $T_{0}$ increased linearly with an increase in $v$, suggesting the absence of trapped entanglements. The values of $L$ were then estimated from the slopes of the curves for $5 \mathrm{k}, 10 \mathrm{k}, 20 \mathrm{k}$ and $40 \mathrm{k}$ Tetra-PEG gels. Here, we used $U=1.0 \times 10^{6}\left(\mathrm{~J} \mathrm{~mol}^{-1}\right)$ and $N=57,114,227$ and $454 \mathrm{~g} \mathrm{~mol}^{-1}$ for the $5 \mathrm{k}, 10 \mathrm{k}, 20 \mathrm{k}$ and $40 \mathrm{k}$ Tetra-PEG gels, respectively. ${ }^{30}$ Figure $4 \mathrm{~b}$ shows $L$ for Tetra-PEG gels with different $N$. $L$ increased from 13 to $33 \mathrm{~nm}$ with an increase in $N . R_{0}$ values for virtual network chains with a polymerization degree of $N$, which correspond to $L$ in the original Lake-Thomas model are also shown. The values of $L$ and $R_{0}$ showed similar magnitude and $\mathrm{N}$-dependence, also suggesting the absence of 

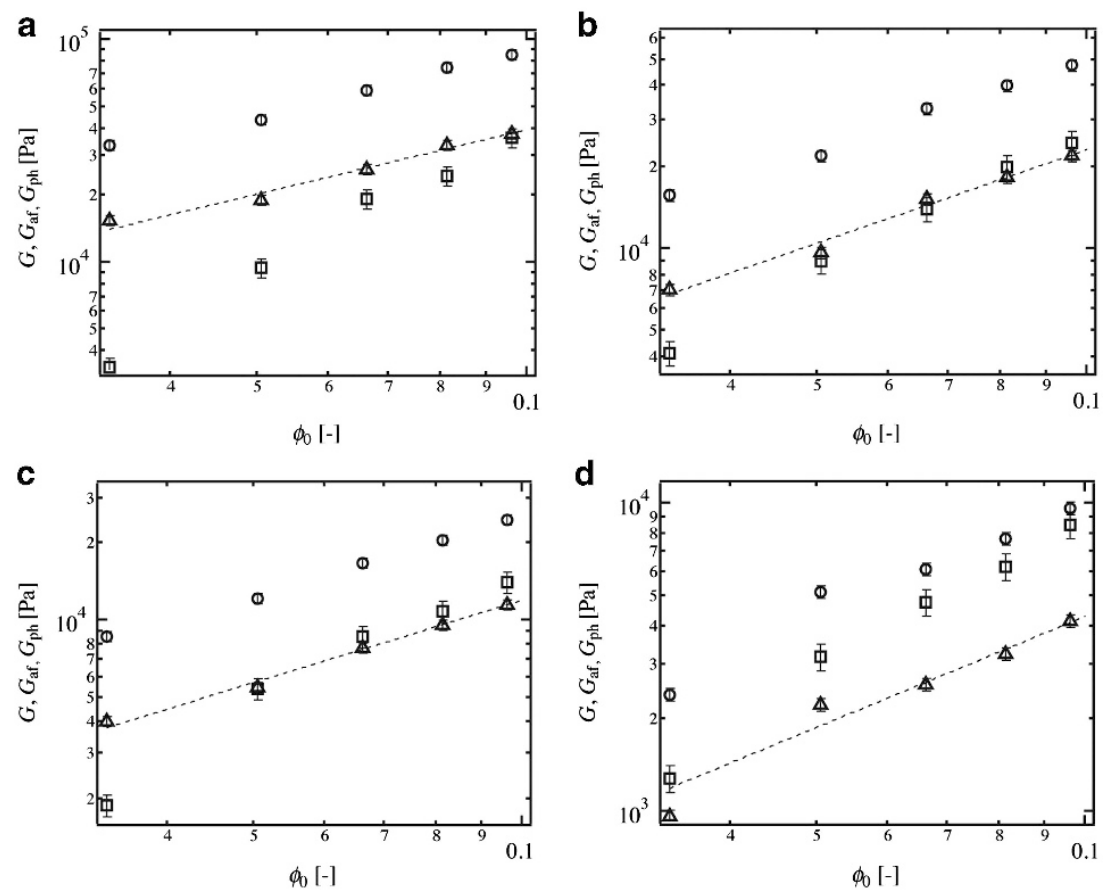

Figure 5 Variation of $G, G_{\mathrm{af}}$ and $G_{\mathrm{ph}}$ as a function of $\phi_{0}$ ((a) $5 \mathrm{k}$, (b) $10 \mathrm{k}$, (c) $20 \mathrm{k}$ (d) $40 \mathrm{k}$ ). The values of $G_{\mathrm{af}}$ and $G_{\mathrm{ph}}$ are calculated from Equations 11 and 12 ( $G$, squares; $G_{\mathrm{af}}$, circles; $G_{\mathrm{ph}}$, triangles; reproduced from Akagi et al. ${ }^{30}$ with permission from the American Chemical Society).

trapped entanglements. From these results, we conclude that there are no appreciable trapped entanglements in Tetra-PEG gels for this experimental condition.

\section{EXAMINATION OF MODELS FOR ELASTIC MODULUS}

In the above sections, we assessed the homogeneity of Tetra-PEG gels. Overall, spatial heterogeneity is highly suppressed and the quantity of trapped entanglements is so small that we can ignore the effect of trapped entanglements on the physical properties. Now, we can calculate the concentration of elastically effective chains simply from the concentration of prepolymers and the reaction conversion using a tree-like approximation (Equations 3-8). In this section, we examine models predicting the elastic modulus, which is one of the simplest physical properties of polymer gels.

There are three popular models predicting the elastic modulus: the affine, ${ }^{27}$ phantom $^{34}$ and junction affine ${ }^{35}$ network models.

$$
\begin{aligned}
G_{a f} & =v k_{\mathrm{B}} T \text { (Affine network model) } \\
G_{p h} & =(v-\mu) k_{\mathrm{B}} T \text { (Phantom network model) }
\end{aligned}
$$

The differences between these models derive from assumptions regarding the fluctuation of cross-links. The affine model assumes that cross-links are firmly connected to the macroscopic body and that network strands deform in the same manner as the macroscopic deformation. However, the phantom network model assumes that cross-links fluctuate and that the deformation of the network strands is suppressed by this fluctuation. The junction affine model is an intermediate between these two models. For a perfect tetra-functional polymer network, the prediction of the affine network model is two times larger than that of the phantom network model. Despite this discrepancy, we do not know the required conditions or even the validity of each model.

To examine these models, we performed tensile testing on the $5 \mathrm{k}$, $10 \mathrm{k}, 20 \mathrm{k}$ and $40 \mathrm{k}$ Tetra-PEG gels and estimated the elastic modulus

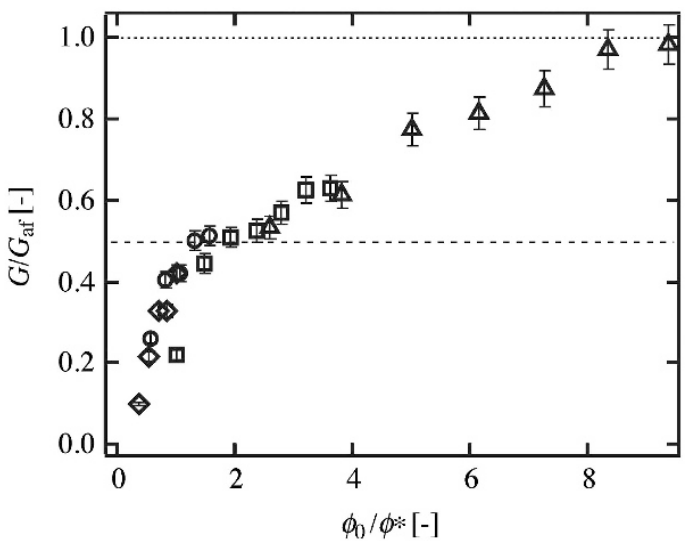

Figure 6 Normalized elastic modulus $\left(G / G_{\mathrm{af}}\right)$ as a function of the normalized polymer volume fraction $\left(\phi_{0} / \phi^{*}\right)(5 \mathrm{k}$, rhombus; $10 \mathrm{k}$, circle; $20 \mathrm{k}$, square; $40 \mathrm{k}$, triangle; reproduced from Akagi et al. ${ }^{30}$ with permission from the American Chemical Society).

$(G)$ from the initial slope of the stress $(\sigma)$-elongation $(\lambda)$ curve. $^{30}$ The variation in $G, G_{\mathrm{af}}$ and $G_{\mathrm{ph}}$ was plotted against $\phi_{0}$ in Figure 5, where $G_{\text {af }}$ and $G_{\text {ph }}$ were calculated from Equations 11 and $12 . G_{\text {af }}$ and $G_{\mathrm{ph}}$ increased linearly with increasing $\phi_{0}$, reflecting the constant $p$. For the 10k and 20k Tetra-PEG gels, good correlation between $G_{\mathrm{ph}}$ and $G$ was observed over a wider range than for the other gels. In the higher concentration region, however, $G$ was slightly larger than $G_{\mathrm{ph}}$. For the $5 \mathrm{k}$ Tetra-PEG gel, the downward deviation of $G$ from $G_{\mathrm{ph}}$ was increasingly pronounced with decreasing $\phi_{0}$. However, $G$ seemed to approach $G_{\mathrm{ph}}$ asymptotically in the high- $\phi_{0}$ region. The $40 \mathrm{k}$ TetraPEG gel showed distinct behavior, as $G$ was above $G_{\text {ph }}$ and close to $G_{\text {af. }}$ Taken together, phantom-like behavior was observed for the condition of low $M_{\mathrm{n}}$ and $\phi_{0}$, whereas affine-like behavior was observed for high $M_{\mathrm{n}}$ and $\phi_{0}$. 
The above trend implies the importance of considering the overlapping polymer volume fraction of prepolymers $\left(\phi^{*}\right)$. Thus, we plotted $G / G_{\text {af }}$ against $\phi_{0} / \phi^{\star}$ (Figure 6). In this figure, phantom and affine network model predictions are the flat lines showing $G / G_{\mathrm{af}}=0.5$ (dashed line) and 1 (dotted line), respectively. All of the data fall onto a single curve, suggesting the validity of the normalization by $\phi^{*}$. In the range from $\phi^{*}$ to $3.0 \phi^{*}$, the elastic moduli are well predicted by the phantom network model. The downward deviation below $\phi^{*}$ implies the formation of elastically ineffective loops $\left(G / G_{\mathrm{af}} \sim\left(\phi_{0} / \phi^{*}\right)^{2.0}\right) .{ }^{36}$ However, in the range above 3.0 $\phi^{*}, G /$ $G_{\mathrm{af}}$ increased with increasing $\phi_{0}$ as $G / G_{\mathrm{af}} \sim\left(\phi_{0} / \phi^{*}\right)^{0.5}$ and approached 1.0. Typically, an increase in $G / G_{\mathrm{af}}$ with an increase in $\phi_{0}$ can be attributed to the introduction of trapped entanglements. However, trapped entanglements are negligible in Tetra-PEG gels. Thus, these data indicate that the model shifts from the phantom to the affine network model with an increase in $\phi_{0}$. This shift is likely owing to the fluctuation of crosslinking points being suppressed by the increased polymer networks.

\section{CONCLUSIONS}

In this review, we presented the results of small angle neutron scattering, infrared, nuclear magnetic resonance and tearing experiments on Tetra-PEG gels with tuned molecular weight of network strands and polymer volume fraction. Through the analyses, we concluded that Tetra-PEG gels formed at the optimal conditions have extremely suppressed heterogeneity and can be treated as near-ideal polymer networks. To our knowledge, this is the first experimental verification of homogeneity in polymer gels. Then, using Tetra-PEG gels as a model system, we observed for the first time a shift in the elastic modulus from the phantom to the affine network models with an increase in polymer volume fraction. Tetra-PEG gels will be useful as a model system for the examination of models and theories predicting other physical properties of polymer gels.

\section{CONFLICT OF INTEREST}

The authors declare no conflict of interest.

\section{ACKNOWLEDGEMENTS}

This work was supported by the Japan Society for the Promotion of Science (JSPS) through the Grants-in-Aid for Scientific Research, the Center for Medical System Innovation (CMSI), and Grant-in-Aids for Scientific Research from the Ministry of Education, Culture, Sports, Science and Technology (no. 23700555).

1 Bastide, J. \& Leibler, L. Large-scale heterogeneities in randomly cross-linked networks Macromolecules 21, 2647-2649 (1988).

2 Mendes, E., Oeser, R., Hayes, C., Boue, F. \& Bastide, J. Small-angle neutron scattering study of swollen elongated gels: butterfly patterns. Macromolecules 29, 5574-5584 (1996).

3 Shibayama, M., Shirotani, Y. \& Shiwa, Y. Static inhomogeneities and dynamics of swollen and reactor-batch polymer gels. J. Chem. Phys. 112, 442-449 (2000).

4 Dusek, K. \& Vojta, V. Concentration of elastically active network chains and cyclization in networks obtained by alternating stepwise polyaddition. B. Polym. J. 9, 164-171 (1977).

5 Shibayama, M. Spatial inhomogeneity and dynamic fluctuations of polymer gels Macromol. Chem. Phys. 199, 1-30 (1998).

6 Rubinstein, M. \& Panyukov, S. Elasticity of polymer networks. Macromolecules 35 6670-6686 (2002)

7 Gottlieb, M., Macosko, C. W., Benjamin, G. S., Meyers, K. O. \& Merrill, E. W. Equilibrium modulus of model poly(dimethylsiloxane) networks. Macromolecules 14 1039-1046 (1981).
8 Mark, J. E. \& Sullivan, J. L. Model networks of end-linked polydimethylsiloxane chains 1. Comparisons between experimental and theoretical values of elastic-modulus and equilibrium degree of swelling. J. Chem. Phys. 66, 1006-1011 (1977).

9 Patel, S. K., Malone, S., Cohen, C., Gillmor, J. R. \& Colby, R. H. Elastic-modulus and equilibrium swelling of poly(dimethylsiloxane) networks. Macromolecules 25 , $5241-5251$ (1992).

10 Michalke, W., Lang, M., Kreitmeier, S. \& Goritz, D. Comparison of topological properties between end-linked and statistically cross-linked polymer networks. J. Chem. Phys. 117, 6300-6307 (2002).

11 Shibayama, M., Takahashi, H. \& Nomura, S. Small-angle neutron-scattering study on end-linked poly(tetrahydrofuran) networks. Macromolecules 28, 6860-6864 (1995).

12 Akagi, Y., Gong, J. P., Chung, U. \& Sakai, T. Transition between phantom and affine network model observed in polymer gels with controlled network structure. Macromolecules 46, 1035-1040 (2013).

13 Akagi, Y., Katashima, T., Katsumoto, Y., Fujii, K., Matsunaga, T., Chung, U., Shibayama, M. \& Sakai, T. Examination of the theories of rubber elasticity using an ideal polymer network. Macromolecules 44, 5817-5821 (2011).

14 Akagi, Y., Katashima, T., Sakurai, H., Chung, U. \& Sakai, T. Ultimate elongation of polymer gels with controlled network structure. RSC Adv. 3, 13251-13258 (2013)

15 Akagi, Y., Matsunaga, T., Shibayama, M., Chung, U. \& Sakai, T. Evaluation of topological defects in Tetra-PEG gels. Macromolecules 43, 488-493 (2010).

16 Katashima, T., Urayama, K., Chung, U. I. \& Sakai, T. Strain energy density function of a near-ideal polymer network estimated by biaxial deformation of Tetra-PEG gel. Soft Matter 8, 8217-8222 (2012).

17 Kurakazu, M., Katashima, T., Chijiishi, M., Nishi, K., Akagi, Y., Matsunaga, T., Shibayama, M. Chung, U. \& Sakai, T. Evaluation of gelation kinetics of Tetra-PEG gel. Macromolecules 43, 3935-3940 (2010).

18 Nishi, K., Chijiishi, M., Katsumoto, Y., Nakao, T., Fujii, K., Chung, U., Sakai, T. \& Shibayama, M. Rubber elasticity for incomplete polymer networks. J. Chem. Phys. 137, 224903 (2012)

19 Nishi, K., Fujii, K., Chijiishi, M., Katsumoto, Y., Chung, U., Sakai, T. \& Shibayama, M Kinetic study for AB-type coupling reaction of tetra-arm polymers. Macromolecules 45, 1031-1036 (2012).

20 Sakai, T., Kurakazu, M., Akagi, Y., Shibayama, M. \& Chung, U. Effect of swelling and deswelling on the elasticity of polymer networks in the dilute to semi-dilute region. Soft Matter 8, 2730-2736 (2012).

21 Sakai, T., Matsunaga, T., Yamamoto, Y., Ito, C., Yoshida, R., Suzuki, S., Sasaki, S. Shibayama., M. \& Chung, U. Design and fabrication of a high-strength hydroge with ideally homogeneous network structure from tetrahedron-like macromonomers. Macromolecules 41, 5379-5384 (2008).

22 Ornstein, L. S. \& Zernike, F. Acculental deviations of density and opalescence at the critical point of a simple substance. Proc. Acad. Sci. (Amsterdam) 17, 793-806 (1914)

23 Shibayama, M., Isono, K., Okabe, S., Karino, T. \& Nagao, M. SANS study on pressureinduced phase separation of poly( $\mathrm{N}$-isopropylacrylamide) aqueous solutions and gels. Macromolecules 37, 2909-2918 (2004).

24 Matsunaga, T., Sakai, T., Akagi, Y., Chung, U. \& Shibayama, M. Structure characterization of Tetra-PEG gel by small-angle neutron scattering. Macromolecules 42, 1344-1351 (2009).

25 Matsunaga, T., Sakai, T., Akagi, Y., Chung, U. I. \& Shibayama, M. SANS and SLS studies on tetra-arm PEG gels in As-prepared and swollen states. Macromolecules 42, 6245-6252 (2009)

26 Lange, F., Schwenke, K., Kurakazu, M., Akagi, Y., Chung, U., Lane, M., Sommer, J., Sakai, T. \& Saalwaechter, K. Connectivity and structural defects in model hydrogels: a combined proton NMR and Monte Carlo simulation study. Macromolecules 44, 9666-9674 (2011).

27 Flory, P. J. Principles of Polymer Chemistry (Cornell University Press, Ithaca, London, 1953)

28 Treloar, L. R. G. The Physics of Rubber Elasticity (Clarendon, Oxford, U.K., 1975).

29 Miller, D. R. \& Macosko, C. W. New derivation of post gel properties of network polymers. Macromolecules 9, 206-211 (1976).

30 Akagi, Y., Gong, J. P., Chung, U. \& Sakai, T. Transition between phantom and affine network model observed in polymer network with controlled network structure. Macromolecules 46, 1035-1040 (2013).

31 Akagi, Y., Sakurai, H., Gong, J. P., Chung, U. \& Sakai, T. Fracture energy of polymer gels with controlled network structures. J. Chem. Phys. 139, 144905 (2013)

32 Liang, S. M., Flu, J., Wu, Z. L., Kurokawa, T. \& Gong, J. P. Toughness enhancement and stick-slip tearing of double-network hydrogels in poly(ethylene glycol) solution. Macromolecules 45, 4758-4763 (2012).

33 Lake, G. J. \& Thomas, A. G. Strength of highly elastic materials. Proc. R. Soc. Lond. A 300, 108-10 (1967)

34 James, H. M. \& Guth, E. Statistical thermodynamics of rubber elasticity. J. Chem Phys. 21, 1039-1049 (1953)

35 Flory, P. J. Theory of elasticity of polymer networks - effect of local constraints on junctions. J. Chem. Phys. 66, 5720-5729 (1977).

36 Vasiliev, V. G., Rogovina, L. Z. \& Slonimsky, G. L. Dependence of properties of swollen and dry polymer networks on the conditions of their formation in solution. Polymer $\mathbf{2 6}$, 1667-1676 (1985). 


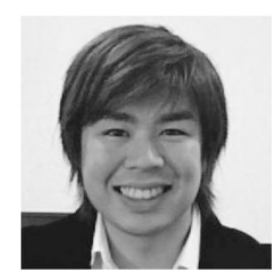

Takamasa Sakai received his PhD in Engineering at the University of Tokyo. His doctoral research with Dr Ryo Yoshida focused on analyzing the kinetics of the Belousov-Zhabotinsky reaction in polymer gels and on developing a novel self-oscillating gel system. He received the best poster presentation award in Gel Symposium 2005. He is currently an assistant professor at the University of Tokyo and is investigating on polymer gels with controlled network structure named Tetra-PEG gel. He focuses on the basics of polymer gels including the mechanical properties and diffusion of substances in polymer gels. 